\title{
Fibrolamellar Hepatocarcinoma: Clinical, Radiologic, and Pathologic Features
}

\author{
Isaac R. Francis, ${ }^{1}$ Farooq P. Agha, ${ }^{1}$ Norman W. Thompson, ${ }^{2}$ and David F. Keren ${ }^{3}$ \\ Departments of ${ }^{1}$ Radiology, ${ }^{2}$ Surgery, and ${ }^{3}$ Pathology, University of Michigan Hospitals, Ann Arbor, Michigan, USA
}

\begin{abstract}
Three new cases of an unusual subtype of hepatocellular carcinoma (HCC) referred to as fibrolamellar hepatocarcinoma (FLHC) recently seen at our institution are described. This report focuses on the clinical, radiologic, and pathologic features of this rare subset of HCC. All three patients were under 30 years of age with no previous history of hepatitis or cirrhosis. Each had had subacute symptoms for 5 months to 1 year before medical attention was sought and/or diagnosis of FLHC was established. There was no reliable correlation with oral contraceptive use in the 2 female patients. Serum alpha-1-fetoprotein levels were normal with only mild elevation of liver enzymes. The CT features, although not specific, were suggestive of an aggressive tumor with amorphous calcification in 2 of the 3 cases. Angiographically all tumors were hypervascular without any evidence of arterioportal shunting or venous invasion of major vessels. The clinical and radiologic recognition of these tumors is important since the surgical resectibility rate and 2- and 5-year survival rates are higher than those applicable to conventional HCC.
\end{abstract}

Key words: Liver tumor - Hepatoma - Hepatocellular carcinoma - Fibrolamellar hepatocarcinoma.

Hepatocellular carcinoma $(\mathrm{HCC})$ is a relatively rare hepatic neoplasm in the western hemisphere, occurring with an incidence of $1: 100,000$, predominantly in men over 50 years of age. The duration of symptoms is short and prognosis usually poor,

Address reprint requests to: Farooq P. Agha, M.D., Department of Radiology Box 13, University Hospital, 1405 East Ann Street, Ann Arbor, MI 48109, USA with mean survival measured in months only. A distinct subtype of $\mathrm{HCC}$ has been recently classified as fibrolamellar hepatocarcinoma (FLHC) due to its characteristic clinical and pathologic features [1-9]. We report 3 newly diagnosed cases of FLHC at our institution during the past $2^{1 / 2}$ years. The analysis of clinical, radiologic, and pathologic features with surgical management and follow-up from 15-33 months in this group of patients constitutes the basis of this report.

\section{Case Reports}

\section{Case 1}

A 22-year-old white woman presented with a 3-day history of right upper quadrant pain associated with nausea, vomiting, and a 44-kg weight loss. There was no present or past history of oral contraceptive use. Physical examination revealed a tender mass in the right upper quadrant with a venous hum. The serum bilirubin and alpha-1-fetoprotein levels were normal with a mild elevation of liver enzymes. CT scan of the abdomen demonstrated (Fig. 1 A) a large mass involving most of the right lobe of the liver and a separate large mass near the porta hepatis compressing the second part of the duodenum. Areas of amorphous calcifications were present within the tumor mass. Hepatic angiography demonstrated a large hypervascular mass in the right lobe of the liver without arteriovenous shunting or venous invasion (Fig. $1 \mathrm{~B}$ ) and a large hypervascular mass in the region just below the porta hepatis. At surgery, a large inoperable mass confined to the right lobe of the liver and a single large metastatic node posterior to the porta hepatis were found compressing the duodenum. Extrahepatic spread precluded right hepatectomy. A liver biopsy and excisional lymph node biopsy were performed. The histopathologic diagnosis was FLHC. Postoperatively she received chemotherapy consisting of adriamycin, mitomycin $\mathrm{C}$, and 5-fluorouracil. Reevaluation 10 months after initial surgery revealed that tumor was confined to the liver; therefore, she underwent an elective right lobe hepatectomy and excision of porta hepatis nodes. She did well until a repeat exploratory laparotomy revealed recurrent tumor in the liver and porta hepatis. Thirtythree months after initial diagnosis, the patient is still alive with recurrent disease. 

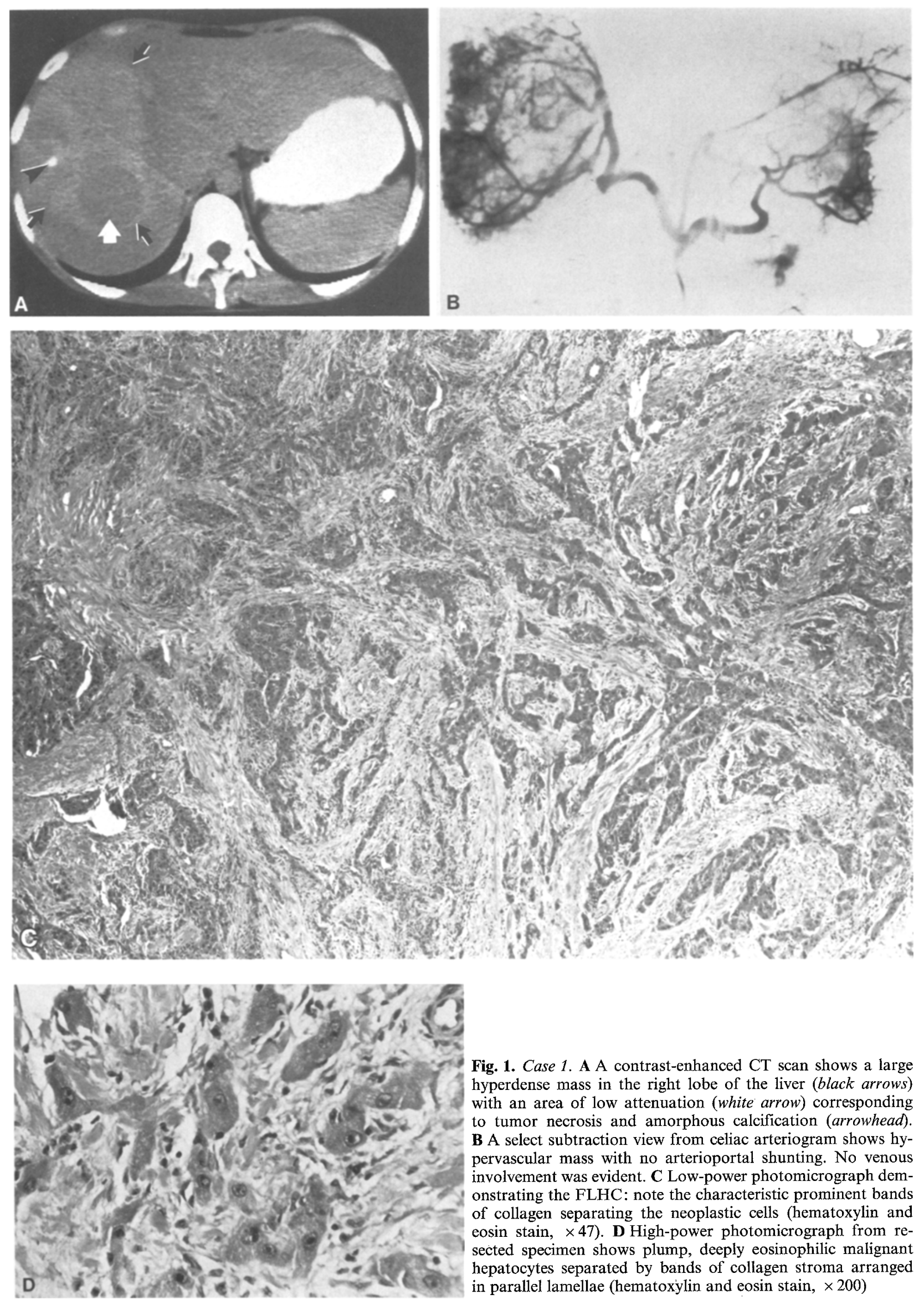

Fig. 1. Case 1. A A contrast-enhanced CT scan shows a large hyperdense mass in the right lobe of the liver (black arrows) with an area of low attenuation (white arrow) corresponding to tumor necrosis and amorphous calcification (arrowhead). B A select subtraction view from celiac arteriogram shows hypervascular mass with no arterioportal shunting. No venous involvement was evident. C Low-power photomicrograph demonstrating the FLHC: note the characteristic prominent bands of collagen separating the neoplastic cells (hematoxylin and eosin stain, $\times 47)$. D High-power photomicrograph from resected specimen shows plump, deeply eosinophilic malignant hepatocytes separated by bands of collagen stroma arranged in parallel lamellae (hematoxylin and eosin stain, $\times 200$ ) 

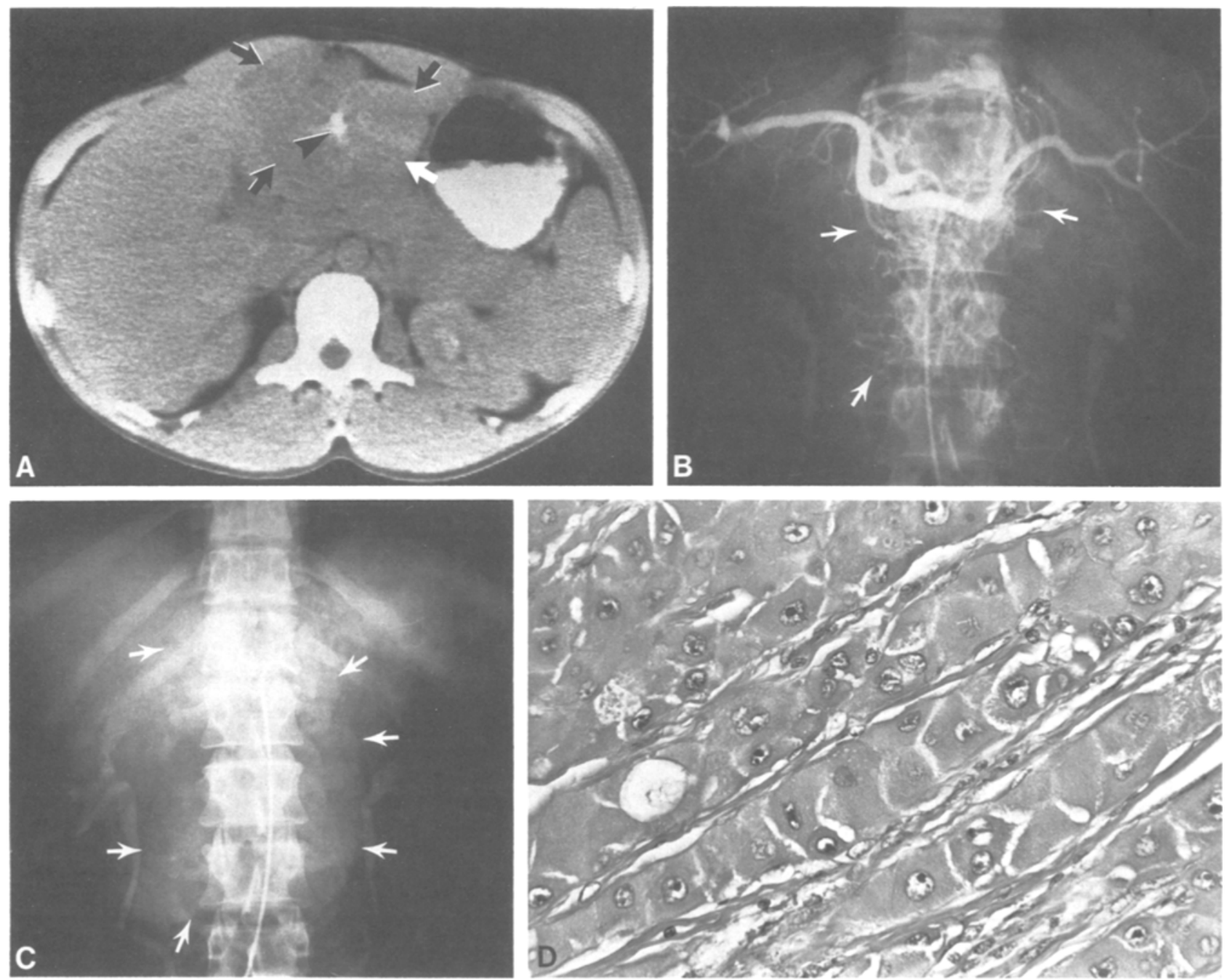

Fig. 2. Case 2. A A postcontrast CT scan shows a large hypodense mass in the left lobe of the liver (arrows) with an area of amorphous calcification (arrowhead). B A selected view from celiac arteriogram shows hypervascular mass occupying the entire left lobe (arrows). C Venous phase (arrows outline the mass). D High-power photomicrograph from the resected specimen showing separation of neoplastic hepatocytes by bands of collagen arranged in parallel lamellae (hematoxylin and eosin stain, $\times 275$ )

\section{Case 2}

A 21-year-old white man first noticed a gradually enlarging mass in the epigastrium for 1 year, and 1 month prior to admission developed nausea and vomiting. He had otherwise been in excellent health. Physical examination revealed a firm, nontender mass. The serum bilirubin and alpha-1-fetoprotein levels were normal with slightly elevated levels of liver enzymes. A postcontrast $C T$ scan demonstrated a large hypodense mass involving the entire left lobe of the liver with focal areas of amorphous calcification (Fig. 2A). Angiography revealed a hypervascular mass occupying the entire left lobe of the liver with no evidence of arteriovenous shunting or venous invasion (Fig. 2B, C). A diagnosis of hepatoma was made and, at this time, a left hepatic lobectomy was performed. The histopathologic diagnosis was FLHC. Metastatic carcinoma was found in one of several lymph nodes. Fifteen months following initial resection, recurrent tumor was detected on a follow-up CT scan. The recurrent tumor, a nodule in the omentum, and 2 involved periaortic lymph nodes were resected. Twenty-one months after initial diagnosis, the patient is alive and not receiving any chemotherapy.

\section{Case 3}

A 27-year-old white woman had a history of intermittent nausea, vomiting, upper abdominal pain, and early satiety for about 1 year for which she was treated with antacids and Cimetadine without any relief. She had used oral contraceptives for 18 months, but had discontinued their use 2 months prior to admission. Physical examination revealed a hard, fixed, nontender epigastric mass continuous with the liver. Serum bilirubin and alpha-1-fetoprotein levels were normal, and liver enzyme levels were mildly elevated. CT scan demonstrated a large inhomogeneous mass involving the entire right lobe, the caudate lobe, and the medial segment of the left lobe (Fig. 3 A). In addition there was extensive retrocaval and paracaval adenopathy with thrombus in the inferior vena cava. Angiography revealed a large hypervascular mass without arteriovenous shunting or invasion of venous structures (Fig. 3B). Exploratory laparotomy showed extensive retroperitoneal lymphadenopathy with encasement of inferior vena cava that precluded curative resection. Several large lymph nodes were present in the porta hepatis, 1 of which was excised for pathologic examination (Fig. 3C). Postoperatively, the patient refused chemo- 

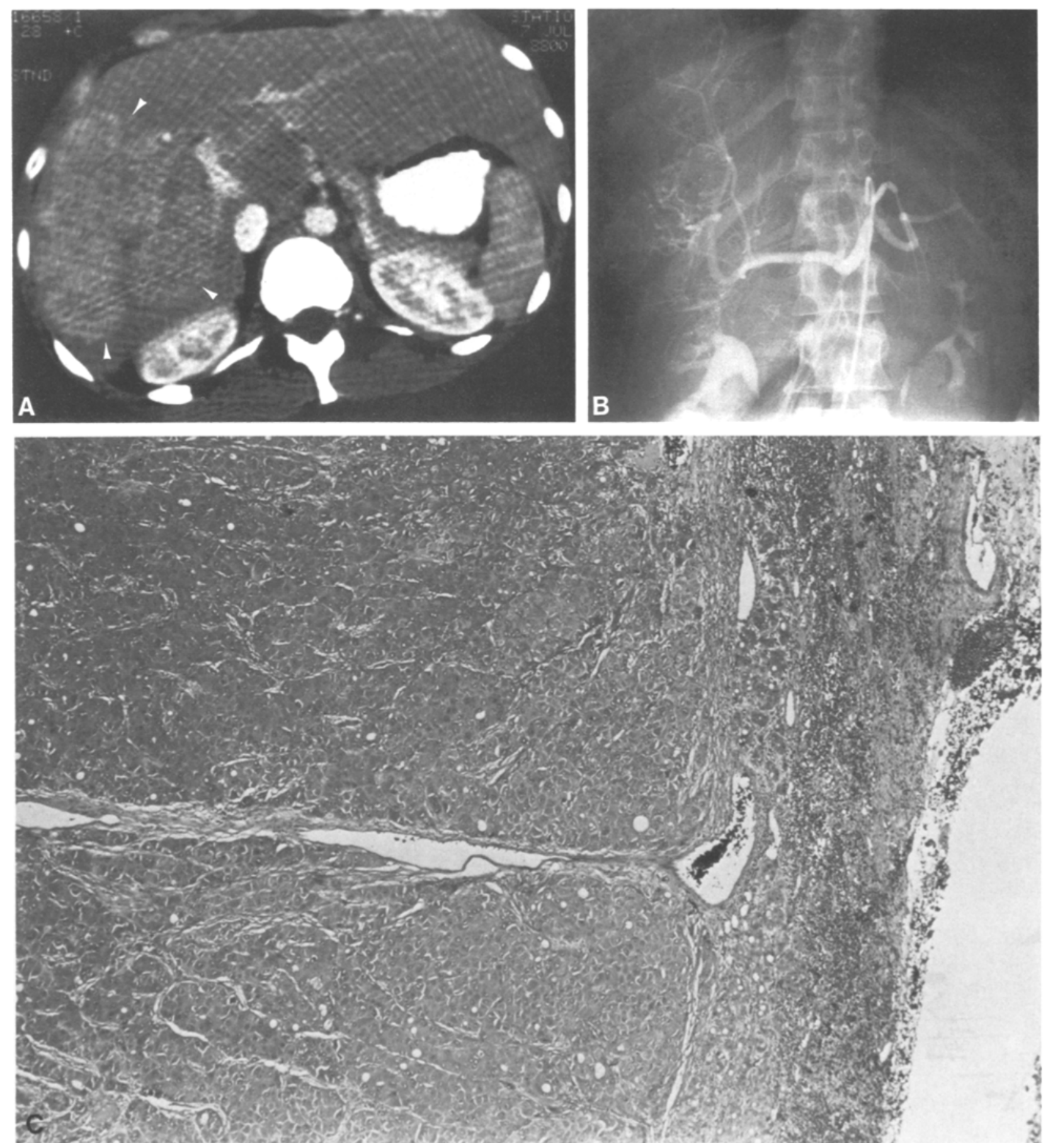

Fig. 3. Case 3. A CT scan during a bolus injection of intravenous iodinated contrast medium shows a large hypervascular mass involving most of the right lobe of the liver (arrowheads). No calcification is seen. B A select view from hepatic arteriogram shows a hypervascular mass without any arterioportal shunting. There was no venous involvement. C Low-power photomicrograph from excised metastatic lymph node from the porta hepatis shows that the nodal morphology at the periphery of the tissue is compressed by the FLHC. Most of this lymph node has been replaced by the malignant hepatocytes (hematoxylin and eosin stain, $\times 47$ )

therapy. Fifteen months following the original diagnosis, she is still alive.

\section{Discussion}

The clinical, radiologic, and pathologic features of HCC have been well-documented previously
[10-14]. Recently, many investigators have described CT findings of HCC [15-23]. Only 1 publication describing a case of FLHC has appeared in the radiologic literature [9]. This variant of $\mathrm{HCC}$ was first described in 1956 by Edmondson [24], who suggested that it might have a relatively favorable prognosis. There was, however, little clinical interest in this tumor until 1980 , when several re- 
ports emphasized its occurrence in young patients and its better prognosis than HCC $[1,2]$.

Other names used for this tumor include eosinophilic carcinoma with lamellar fibrosis [10], polygonal cell type with fibrous stroma $[2,11]$, scirrhous hepatoma $[25,26]$, and fibrolamellar oncocytic hepatoma $[3,5]$.

The clinical features of FLHC are distinctly different from those of other types of HCC. FLHC is predominantly a disease of young adults and shows no sex predilection, in contrast to other types of HCC which occur in older men, usually 50-70 years of age, with preexisting cirrhosis. The mean age of patients affected by FLHC varies from 25 to 26.4 in larger series $[1,2,5]$. Our three patients had a mean age of 23.3 years. This younger age distribution approximates the age of patients with benign liver tumors such as focal nodular hyperplasia and hepatic adenomas [11].

The latter 2 lesions have been found in association with the use of oral contraceptives but few documented reports of hepatocellular carcinoma have been described in women using oral contraceptives $[27,28]$. Although speculation persists regarding the premalignant nature of lesions induced by oral contraceptives, no substantive morphologic evidence supports malignant transformation of focal nodular hyperplasia or hepatic adenomas [29, 30]. Although 1 of our 3 patients had used oral contraceptive pills, it is most likely that the occurrence of FLHC was coincidental, since half of the reported cases have been in men. Recently an important etiologic association between alpha-1-antitrypsin deficiency due to homozygous protease inhibitor $(\mathrm{Pi}) \mathrm{ZZ}$ and $\mathrm{HCC}$, including its subset fibrolamellar type, has been reported [31, 32].

Nausea, vomiting, upper abdominal pain, and occasional weight loss are the predominant symptoms. The duration of symptoms in our series was 4-12 months. The tumor is usually palpable in association with the liver. The laboratory studies show mild derangement of liver function tests. Alpha-1-fetoprotein levels may be normal, as occurred in all 3 of our patients. Recently high serum vitamin-B12-binding capacity as a marker of FLHC [33, 34] and abnormally elevated copper and copper binding protein in malignant hepatocytes have also been reported in FLHC [35].

Computed tomographic evaluation usually reveals a large solitary tumor mass involving an entire lobe or multicentric involvement with amorphous calcifications. The assessment of tumor vascularity in our series, by CT, was not possible since scans in 2 of 3 patients were not performed by dynamic CT following bolus injection of iodinated intravenous contrast medium. In our patients, the right lobe was involved in 2 and the left lobe in the 3rd patient; predominant involvement of the left lobe has been reported in larger series $[1,2]$. CT findings revealed no specific characteristic features to differentiate this entity from other benign or malignant tumors of the liver. CT has been useful in demonstrating local and regional metastatic spread, determining response to chemotherapy, and detecting recurrence after surgery. Angiographically, the tumor is hypervascular with no arteriovenous shunting or venous involvement; these features are usually seen in a conventional HCC.

Pathologically the tumor exhibits two characteristic features: deep eosinophilic plump hepatocytes and abundant fibrous stroma arranged in parallel bands. Fibrosis is distinctly unusual in HCC and only occurs in a minority of patients. In published reports, the surgical resectibility rate for $\mathrm{HCC}$ varies from 9 to $23 \%$ with an average of $17 \%$, which contrasts sharply with the $48 \%$ resectibility rate reported in FLHC. Evidence for prolonged survival (mean 32-68 months) for patients with FLHC is impressive [36] compared to 6 months (5.9-7 months) in HCC [2]. The average survival rate in our three patients is 23 months (1533 months). All of our patients are still alive at the time of this report. Increased awareness and a high index of suspicion of FLHC, despite the nonspecific CT features, are essential for early diagnosis of this unusual variant of HCC. Higher resectibility rate and prolonged survival justify aggressive surgical management, including resection of metastases.

Acknowledgement. The authors gratefully acknowledge the excellent secretarial assistance of Ms. Marilyn Campeau, Ms. Sandra Ressler, and Ms. Barbara Smith.

\section{References}

1. Craig JR, Peters RL, Edmundson HA, Omata M: Fibrolamellar carcinoma of liver: a tumor of adolescents and young adults with distinctive clinico-pathologic features. Cancer $46: 372-379,1980$

2. Berman MM, Libby NP, Foster JH: Hepatocellular carcinoma: polygonal cell type with fibrous stroma - an atypical variant with a favorable prognosis. Cancer $46: 1448-1455$, 1980

3. Fahri DC, Shikes RH, Silverberg SG: Ultrastructure of fibro lamellar oncocytic hepatoma. Cancer 50:702-709, 1980

4. Lefkowitch JH, Arborgh BAM, Scheuer PJ: Oxyphilic granular hepatocytes: mitochondrion rich liver cells in hepatic disease. Am J Clin Pathol 74:432-441, 1980

5. Fahri DC, Shikes RH, Murari PJ, Silverberg SJ : Hepatocellular carcinoma in young adults. Cancer 52:1516-1525, 1983

6. Lack EE, Neave C, Vawter GF : Hepatocellular carcinoma: review of 32 cases in childhood and adolescent. Cancer $52: 1510-1515,1983$

7. Stromeyer FW, Ishak KG, Gerber MA: Ground glass cells in hepatocellular carcinoma. Am J Clin Pathol 74:254-258, 1980 
8. Albukerk $\mathrm{J}$ : Hepatocellular carcinoma in young adults. $N Y$ State J Med 81:1341-1344, 1981

9. Wang LK, Link DP, Frey CF, Ruebner BH, Tesluk H, Primstone NR: Fibrolamellar hepatocarcinoma: radiology, management and pathology. AJR 139:172-175, 1982

10. Peters RL: Pathology of hepatocellular carcinoma. In Okuda K, Peters RL (eds): Hepatocellular Carcinoma. New York: John Wiley and Sons, 1976

11. Foster JH, Berman MM: Solid liver tumors. In Ebert PA (ed): Major Problems in Clinical Surgery, vol 22. Philadelphia: WB Saunders, 1977, pp 5-8, 86-97, 111-128

12. Edmondson HA: Tumors of liver and intrahepatic bile ducts. In: Atlas of Tumor Pathology, section 7, fascicle 2. Washington DC: Armed Forces Institute of Pathology, 1958

13. Okuda K, Musha H, Yoshida T, Kanda Y, Yamazaki T, Jinnouchi S, Moriyama M, Kawaguchi S, Kubi Y, Shimokawa Y, Kojiro M, Kuratomi S, Sakamoto K, Nakashima $\mathrm{T}$ : Demonstration of growing cast of hepatocellular carcinoma in the portal vein by celiac angiography: "the thread and streaks sign." Radiology 117:303-309, 1975

14. Reuter SR, Redman HC, Siders DB: The spectrum of angiographic findings in hepatoma. Radiology $94: 89-94,1970$

15. Kunstlinger F, Federle MP, Moss AA, Marks W: Computed tomography of hepatocellular carcinoma. $A J R$ $134: 431-437,1980$

16. Itai $Y$, Nishikawa J, Tasaka A: Computed tomography in the evaluation of hepatocellular carcinoma. Radiology $13: 165-170,1979$

17. Hosoki T, Chatani M, Mori S: Dynamic computed tomography of hepatocellular carcinoma. AJR 139:1099-1106, 1982

18. Takashima T, Matsui O, Suzuki M, Ida M: Diagnosis and screening of small hepatocellular carcinoma. Radiology $145: 635-638,1980$

19. Matsui O, Kadoya M, Suzuki M, Inoue K, Ida H, Ida M, Takashima T: Dynamic sequential computed tomography during arterial portography in the detection of hepatic neoplasms. Radiology 146:721-727, 1983

20. Bernardino M, Lewis E: Imaging hepatic neoplasms. Cancer 50:2666-2671, 1982

21. Inamoto K, Sugiki K, Yamasaki H, Nakao N, Takashi $\mathrm{M}$ : Computed tomography and angiography of hepatocellular carcinoma. J Comput Assist Tomogr 4:832-839, 1980

22. Dunnick NR, Ihde DC, Dopman JL, Richard-Bates H: Computed tomography in primary hepatocellular carcinoma. J Comput Assist Tomogr 4:59-60, 1980

23. Hosoki T, Toyonaga Y, Araki Y, Mori S: Dynamic com- puted tomography of isodense hepatocellular carcinoma. $J$ Comput Assist Tomogr 8:263-268, 1984

24. Edmondson HA : Differential diagnosis of tumor and tumor like lesions of the liver in infancy and childhood. Arch Dis Child 1:168-186, 1956

25. Anthony PP: Primary carcinoma of the liver: study of 282 cases in Uganda Africans. I Pathol 110:37-48, 1973

26. Gibson JR: Histological Typing of Tumors of the Liver, Biliary Tract and Pancreas. Geneva: World Health Organization, 1978

27. Neuberger J, Portmann B, Nunnerley HB, Laws JW, Davis M, William R: Oral-contraceptive-associated liver tumors: occurrence of difficulties in diagnosis. Lancet 1:273-276, 1980

28. Davis M, Portmann B, Searle M, Wright R: Histologic evidence of carcinoma in a hepatic tumor associated with oral contraceptives. Br Med J 4:496-498, 1975

29. Christopherson WM: Possible etiologic factors for some malignant hepatic tumors. In Stroehlein JR, Romsdahl MM (eds): Gastrointestinal Cancer. New York: Raven Press, 1981, pp 257-269

30. Goodman ZP, Ishak KG: Hepatocellular carcinoma in women: probable lack of etiologic association with oral contraceptive steroids. Hepatology 2(4):440-444, 1982

31. Govindarajan S, Ashcaval M, Paters RL: Alpha-1-antitryp$\sin$ phenotypes in hepatocellular carcinoma. Hepatology 1(6):628-631, 1981

32. Vergalla J, Jones EA, Kew MC: Alpha-1-antitrypsin deficiency and hepatocellular carcinoma. $S$ Afr Med $J$ 64:950-951, 1983

33. Paradinas FJ, Melia WM, Wilkinson ML, Portmann B, Johnson PJ, Murray-Lyon IM, Williams R: High serum vitamin $\mathrm{B} 12$ binding capacity as a marker of the fibrolamellar variant of hepatocellular carcinoma. $\mathrm{Br} \mathrm{Med} J$ $285: 840-842,1982$

34. Sheppard KJ, Bradbury DA, Davies J, Ryrie DR: High serum vitamin $\mathrm{B} 12$ binding capacity as a marker of the fibrolamellar variant of hepatocellular carcinoma. $\mathrm{Br} \mathrm{Med}$ $J$ 286(6358):57, 1983

35. Lefkowitch JH, Muschel R, Price JB, Marboe C, Braunhut $\mathrm{S}$ : Copper and copper-binding protein in fibrolamellar liver cell carcinoma. Cancer 51:97-100, 1983

36. Chuong JJ, Livestone EM, Barwick KW: The histologic and clinical indicators of prognosis in hepatoma. $J$ Clin Gastroenterol 4(6):547-552, 1982

Received: October 15, 1984; accepted: December 19, 1984 